\title{
Mesoscopic Fermi gas in a harmonic trap
}

\author{
J. Schneider and H. Wallis \\ Max-Planck-Institut für Quantenoptik, Hans-Kopfermann-Straße 1, D-85748 Garching, Germany
}

(submitted to Phys. Rev. A, February 24, 2018)

\begin{abstract}
We study the thermodynamical properties of a mesoscopic Fermi gas in view of recent possibilities to trap ultracold atoms in a harmonic potential. We focus on the effects of shell closure for finite small atom numbers. The dependence of the chemical potential, the specific heat and the density distribution on particle number and temperature is obtained. Isotropic and anisotropic traps are compared. Possibilities of experimental observations are discussed.
\end{abstract}

\section{INTRODUCTION}

The recent realizations of Bose-Einstein condensation in dilute atomic vapors [1] 3] have not only stimulated many investigations on Bose atoms but also studies of degenerate Fermi gases. As opposed to charged Fermi gases, i.e. nucleons or electrons in solids, the effects of Fermi statistics in neutral atomic gases occur at much lower temperatures (typically below $10^{-7} \mathrm{~K}$ ) and at densities which allow a treatment as dilute quantum gas because of the weak interatomic interactions. Due to the selection rules for collisions, spin-polarized fermionic alkali atoms, like ${ }^{6} \mathrm{Li}$ or ${ }^{40} \mathrm{~K}$ in magnetic traps, remain metastable in the regime of quantum statistical degeneracy. Due to Fermi statistics the lowest scattering channel (s-wave scattering) is closed for atoms in identical magnetic sublevels. Therefore an ultracold spin-polarized Fermi gas will be less influenced by interactions than the ultracold Bose gases [1 3]. Also a BCS transition as studied in [4.5] is naturally excluded here. The only remaining interaction is the magnetic dipole-dipole interaction between the atoms. An estimate of its contribution to the mean-field [6] yields $\left\langle V_{D D}\right\rangle=\left(\hbar a_{d} / M\right)\left\langle r^{-3}\right\rangle$ where $M$ is the atomic mass and $a_{d}<10^{-10} \mathrm{~m}$. This effect is neglected here.

The purpose of the present paper is rather to consider the stationary features of an ideal Fermi gas in isotropic or anisotropic harmonic traps. Since our results are based on the numerical calculation of the state sum without further approximation, they are complementary to the recent paper of Butts and Rokhsar [7], where a continuous spectrum and Thomas-Fermi approximation were used. That treatment becomes exact in the limit of large particle numbers. In contrast, we here focus on the effects of small particle numbers, where the shell structure still affects the behavior of the many-particle system.

The outline of the paper is as follows. In Sec.II we investigate the case of an isotropic harmonic trap. We analyze the influence of the shell structure on the chemical potential and the specific heat as a function of number and temperature. For small particle number, density distributions deviating from the Thomas-Fermi distribution are obtained. In Sec. III the anisotropic trap is considered with respect to chemical potential, specific heat and density. The deviations from the isotropic trap are discussed. In Sec. IV the results are summarized in view of experimental realizations, and the validity of the assumptions underlying our theoretical approach is discussed.

\section{PROPERTIES OF THE FERMI GAS IN AN ISOTROPIC HARMONIC TRAP}

\section{A. Degeneracies and the Fermi edge}

We first study the isotropic harmonic trap with potential energy

$$
V=\frac{M \omega^{2}}{2}\left(x^{2}+y^{2}+z^{2}\right),
$$

and frequency $\omega$, because of its distinct features compared with the anisotropic trap studied below. In this case the degeneracy of states with equal energy

$$
E_{\nu}=(\nu+3 / 2) \hbar \omega
$$

is given by

$$
g_{\nu}=\frac{1}{2}(\nu+1)(\nu+2),
$$

i.e. equal to the number of simple partitions of $\nu$ as a sum of three integers $\nu=\nu_{x}+\nu_{y}+\nu_{z}$. Since $g_{\nu}$ gives the degeneracy of a shell of energy $E_{\nu}$, one finds the total number $S_{\alpha}$ of quantum states with energy smaller than $E_{\alpha}$ as the sum over the shells $0 \leq \nu \leq \alpha$,

$$
\sum_{\nu=0}^{\alpha} g_{\nu}=S_{\alpha}
$$

The sums

$$
S_{\alpha}=\frac{1}{6}(\alpha+1)(\alpha+2)(\alpha+3)
$$

define the sequence $\left\{S_{\alpha}\right\}=\{1,4,10,20,35,56, \ldots\}$ and so forth. Note that each oscillator state is assumed to be filled with a single Fermion since only one spin orientation is confined by the magnetic trap. 
For simplicity, our calculations are done using the grand canonical ensemble [8]. The thermal occupation of a state with energy $E_{\nu}$ at a temperature $k_{B} T=1 / \beta$ is given by Fermi-Dirac statistics as

$$
n_{\nu}=\frac{1}{z^{-1} \exp (\beta \hbar \omega \nu)+1}
$$

where the fugacity $z$ is determined from the condition

$$
\sum_{\nu=0}^{\infty} g_{\nu} n_{\nu}=N
$$

The definition of the fugacity $z=\exp \beta(\mu-(3 / 2) \hbar \omega)$ absorbs the zero-point energy. $N$ is the total number of particles in the trap. For a given particle number one can determine the Fermi energy

$$
E_{F}=\left(\nu_{F}+3 / 2\right) \hbar \omega
$$

where $\nu_{F}$ is the shell up to which the trap levels are filled with particles at temperature $T=0$. In this limit $(\beta \rightarrow \infty)$ the Fermi-Dirac distribution approaches a step function. Note that in the case of a mesoscopic ensemble the zero-temperature equation

$$
\sum_{\nu=0}^{\infty} g_{\nu} \theta\left(\nu_{F}-\nu\right)=N
$$

does not have a solution for each $N$, but only for the discrete set of total particle numbers $N \in\left\{S_{\alpha}\right\}$. For $N \notin\left\{S_{\alpha}\right\}$ we may still define $\nu_{F}=\left\lceil x_{F}\right\rceil$ as the smallest integer equal or greater than the exact solution of Eq. (2.8),

$$
x_{F}=A+\frac{1}{3 A}-2,
$$

where $A$ is given by

$$
A=\left(3 N+\sqrt{9 N^{2}-1 / 27}\right)^{1 / 3} .
$$

The expression $E_{F}=\hbar \omega\left(\left\lceil x_{F}\right\rceil+3 / 2\right)$ has to be compared to the Thomas-Fermi approximation for the Fermi edge,

$$
\tilde{E}_{F}=\hbar \omega(6 N)^{1 / 3} \text {. }
$$

\section{B. Calculation of the chemical potential}

We now turn to the determination of the most important properties of the ideal gas. The fugacity resp. the chemical potential are obtained by a numerical solution of Eq. (2.6) for given temperature and particle number which is exact inasmuch as it does not invoke the Thomas-Fermi approximation. The results are then analyzed in certain limits below. Fig. 1 shows the dependence of the chemical potential $\mu$ on the atom number $N$ for small temperatures. Whereas the solid lines correspond to the Thomas-Fermi approximation, the three other curves were obtained numerically by truncating the sum in Eq. (2.6) at sufficiently high $\nu$. They display a step-like variation that becomes increasingly smoother for higher temperatures. The step function will appear to be broken into smaller steps in the anisotropic oscillator case studied below. Here, the steps occur whenever a shell is saturated and $\nu_{F}$ acquires the next higher integer value. $\mu$ converges to a certain ("plateau") value $\hbar \omega\left(\nu_{F}+3 / 2\right)$ in the limit $T \rightarrow 0$ for all $N$ which do not coincide with a "magic" number $S_{\alpha}$. However, if a shell is closed $\left(N=S_{\nu_{F}}\right), \mu$ takes the value $\mu=\hbar \omega\left(\nu_{F}+2\right)$, which is very close to the value of the Thomas-Fermi result at $S_{\nu_{F}}$ (solid line in Fig. 11). As can be shown by asymptotic expansion, the two curves intersect approximately at $N=S_{\alpha}$ respectively $N=\left(S_{\alpha}+S_{\alpha-1}\right) / 2$, i.e. at total or half filling of shells.

This information is displayed in detail in Fig. 2 giving the dependence of $\mu$ on the temperature around the value $N=S_{7}=120$. At $T=0$, the $N=119$ curve still approaches the previous plateau value $\mu / \hbar \omega=7+3 / 2$, whereas the $N=121$ curve has to approach the value $\mu / \hbar \omega=8+3 / 2$.

The temperature dependence of $\mu$ can be calculated analytically in the limits of high and low temperature. The high temperature region of Fig. 2 is well described by the Sommerfeld-like formula [0]

$$
\tilde{\mu}(T)=\tilde{E}_{F}\left(1-\frac{\pi^{2}}{3}\left(\frac{k T}{\tilde{E}_{F}}\right)^{2}\right)
$$

where the factor $\pi^{2} / 3$ replaces the factor of $\pi^{2} / 12$ of the usual case of fermions in a box. We note that for high temperatures the exact result for $N=119$ approaches the Sommerfeld approximation for $N=120$ (see Fig. 2).

In the low temperature regime, the variation of $\mu$ can be analyzed in analogy to the chemical potential of electrons in an intrinsic semi-conductor. We first consider the "magic" numbers $N \in\left\{S_{\alpha}\right\}$. Let $N_{>}(T)$ be the number of atoms excited to states above $E_{F}$ and $N_{<}(T)$ the number of unoccupied states ("holes") at or below $E_{F}$

$$
\begin{aligned}
& N_{>}(T)=\sum_{\nu=\nu_{F}+1}^{\infty} \frac{g_{\nu}}{z^{-1} \exp (\beta \hbar \omega \nu)+1} \\
& N_{<}(T)=\sum_{\nu=0}^{\nu_{F}} g_{\nu}\left(1-\frac{1}{z^{-1} \exp (\beta \hbar \omega \nu)+1}\right) .
\end{aligned}
$$

For low temperatures, i.e. for

$$
k_{B} T \ll E_{\nu_{F}+1}-\mu \quad \text { and } \quad k_{B} T \ll \mu-E_{F},
$$

the number of "particles" and "holes" can be approximated as

$$
\begin{aligned}
& N_{>}(T) \approx \Sigma_{>} e^{-\beta\left(E_{\nu_{F}+1}-\mu\right)} \\
& N_{<}(T) \approx \Sigma_{<} e^{-\beta\left(\mu-E_{F}\right)}
\end{aligned}
$$


where

$$
\begin{aligned}
& \Sigma_{>}=\sum_{\nu=\nu_{F}+1}^{\infty} g_{\nu} e^{-\beta\left(E_{\nu}-E_{\nu_{F}}+1\right)} \\
& \Sigma_{<}=\sum_{\nu=0}^{\nu_{F}} g_{\nu} e^{-\beta\left(E_{F}-E_{\nu}\right)}
\end{aligned}
$$

are essentially Boltzmann sums. On combination of the above equations one arrives at

$$
N_{>}(T) \cdot N_{<}(T)=\Sigma_{>} \Sigma_{<} e^{-\beta \hbar \omega} .
$$

From this condition the chemical potential can be determined. As for $N \in\left\{S_{\alpha}\right\}$ the Fermi shell is totally filled at $T=0, N_{>}(T)$ must equal $N_{<}(T)$ in that case, and one obtains from Eq. (2.16 2.20) the low temperature behavior

$$
\mu(T)=\hbar \omega\left(\nu_{F}+2\right)-\frac{k_{B} T}{2} \ln \left(\frac{\Sigma_{>}}{\Sigma_{<}}\right) .
$$

Thus $\mu(0)$ lies in the middle of the "gap" between $E_{F}$ and $E_{F}+\hbar \omega$, like in an intrinsic semi-conductor where the valence band is filled at zero temperature and the chemical potential lies in the middle of the bandgap. It shows a slow linear decrease with increasing temperature, governed by the small factor $\ln \left(\Sigma_{>} / \Sigma_{<}\right)$.

If on the other hand $N \notin\left\{S_{\alpha}\right\}$, one can calculate $\mu(T)$ from the following approximation. For very low temperatures the Fermi function is well approximated by $n_{\nu}=1$ for $\nu<\nu_{F}$ resp. $n_{\nu}=0$ for $\nu>\nu_{F}$. The number of occupied states in the Fermi shell $\Delta N=N-S_{\nu_{F}-1}$ then reads approximately

$$
\Delta N=\frac{g_{\nu_{F}}}{z^{-1} \exp \left(\beta \hbar \omega \nu_{F}\right)+1} .
$$

Assuming that $\Delta N$ is a constant for very low temperatures, one can solve Eq. (2.22) for the chemical potential

$$
\mu(T)=\hbar \omega\left(\nu_{F}+\frac{3}{2}\right)-k T \ln \left(\frac{g_{\nu_{F}}}{\Delta N}-1\right) .
$$

This expression varies linearly with $T$ for non-vanishing $\Delta N$, with its slope changing sign at $\Delta N=g_{\nu_{F}} / 2$. If the highest shell is less than half filled $\left(\Delta N<g_{\nu_{F}} / 2\right), \mu(T)$ decreases linearly from $\mu(0)=E_{\nu_{F}}$ - if it is more than half filled, it increases linearly from the $\mu(0)=E_{\nu_{F}}$. The exact result then approaches the Sommerfeld curve. The range of validity $\Delta T$ of the linear approximation can be roughly determined by equating

$$
\frac{\hbar \omega}{2} \equiv k \Delta T \ln \left(g_{F}-1\right)
$$

since the maximum deviation from the Sommerfeld approximation equals $\hbar \omega / 2$ at $\Delta N=1$ (see Fig.2). For $\nu_{F}=7$ one obtains this range as $k_{B} \Delta T / \hbar \omega \leq 0.14$. For larger values of $\Delta N$ the slope is smaller and the validity range may be larger.

\section{Specific heat}

The discontinuity of the chemical potential manifests itself most drastically in the specific heat of the gas. It is calculated from the total energy

$$
U(T)=\sum_{\nu=0}^{\infty} \frac{g_{\nu} \hbar \omega \nu}{z^{-1} \exp (\beta \hbar \omega \nu)+1}
$$

via

$$
C(T)=\frac{\partial U(T)}{\partial T} .
$$

The usual Sommerfeld approximation for low temperatures yields

$$
\frac{\tilde{C}(T)}{N k}=\pi^{2} \frac{k T}{\hbar \omega(6 N)^{1 / 3}} .
$$

whereas the classical high temperature limit equals $C_{c l} / N k=3$. Here, we determine $C(T)$ for finite $N$ from the state sum and compare it to the Sommerfeld approximation. The results are shown in Fig. 3, where the $N^{1 / 3}$ scaling is already included on the ordinate. In the limit of ultra-low temperatures the finite-size effects result in a deviation from the linear Sommerfeld prediction. For higher temperature the specific heat $C(T)$ approaches the Sommerfeld result (2.27).

At very low temperatures $C(T)$ remains zero instead of increasing linearly. This is consistent with the assumptions leading to Eq. (2.23) which are confirmed by the calculation of the state sums. In the ultra-low temperature regime no states above the Fermi energy are populated due to the energy gap, the total energy does not increase and $C(T)$ equals zero. This explanation seems to be correct also for the case of closed shells $(N=84,120,9880)$ where Eq. (2.23) does not hold. Note that the range where $C(T)$ remains zero is the same as the range of validity of the linear approximation for $\mu(T)$.

At intermediate temperatures a strong non-monotonic $N$-dependence of the specific heat at constant $T$ occurs, roughly at those temperatures where the linear approximation ceases to be applicable. The origin of this behavior is revealed in Fig. 4 . Each time a shell closure occurs, $C(N)$ runs through a maximum. At these points, the system can access a new, totally empty shell, at the expense of adding the gap energy to the new particles. On the contrary, the minima occur half way between successive shell closures. In Fig. 5, the total heat capacity is plotted versus $N$ without a rescaling.

Fig. 3 shows another interesting detail: the two limiting curves for totally filled shells $(N=84,120,9880)$ resp. half filled shells $(N=102,142,10270)$ do not depend on $\nu_{F}$. Up to $k_{B} T / \hbar \omega \approx 0.5$ the function $(6 N)^{1 / 3} C /(N k)$ does not seem to depend on $N$ explicitly for the values considered here, but only on the relative filling of the Fermi shell. This is related to the fact that the dependence $\mu(T)$ shown in Fig. 2 repeats itself around each value for $\nu_{F}$. 


\section{Density distributions}

Density distributions in traps can be measured quite easily. In an isotropic oscillator, one expects radially symmetric distributions. The radial wavefunctions $u_{n_{r}, l}$ (cf. e.g. [9]) are numbered by a radial quantum number $n_{r}$ and angular momentum $l$. The corresponding energy is $E_{n_{r}, l}=\hbar \omega\left(2\left(n_{r}-1\right)+l+3 / 2\right)$ so $\nu=2\left(n_{r}-1\right)+l$. To compute the total density one has to sum up the squared wavefunctions weighted correctly with $n_{\nu}$

$$
\rho(r)=\sum_{\nu=0}^{\infty} n_{\nu}(T) \sum_{n_{r}=1}^{[\nu / 2]+1} \frac{2 l+1}{4 \pi}\left|u_{n_{r}, l}(r)\right|^{2}
$$

where $l=\nu-2\left(n_{r}-1\right)$. The factor $(2 l+1) /(4 \pi)$ is due to the summation over all states with $m=-l, \ldots, l$. In Fig. 6, $\rho(r)$ is displayed for different particle numbers and temperatures and scaled with the size of the trap groundstate $\sigma=\sqrt{\hbar /(M \omega)}$. The zero temperature result from the Thomas-Fermi approximation (cf. [7], see Eq. (3.6), $\lambda=1)$ is also shown.

For $N=120$ (closed shell, $\nu_{F}=7$ ), one observes a central minimum that disappears at $N=142$ (half filled shell, $\left.\nu_{F}=8\right)$. This shell gets totally filled at $N=165$ where $\rho(r)$ has a maximum at $r=0$. The $N$-dependence of the density at $r=0$ is due to the fact that shells with odd $\nu$ do not contribute to $\rho(0)$ because they are made up of odd angular momentum states which all have zero density at the origin. The curves for $k_{B} T=0.1 \hbar \omega$ are almost indistinguishable from the $T=0$ curves. The minima and maxima are still visible at $k_{B} T=0.25 \hbar \omega$ but disappear for temperatures above $k_{B} T=\hbar \omega$. For not too high temperatures the density approaches the Thomas-Fermi result. Interestingly, $\rho(r)$ is almost equal to this approximation for half filled Fermi shells.

\section{THE ANISOTROPIC CASE}

\section{A. Chemical potential and heat capacity}

Experimentally realized magnetic traps are usually at least slightly anisotropic. In this section we therefore study a deformed oscillator with a potential

$$
V=\frac{M \omega^{2}}{2}\left(x^{2}+y^{2}+\lambda^{2} z^{2}\right)
$$

i.e. we allow for prolate and oblate ellipsoid iso-energy surfaces. Accordingly, the energy eigenvalues are

$$
E_{\nu_{r}, \nu_{z}}=\hbar \omega\left(\nu_{r}+1+\lambda\left(\nu_{z}+\frac{1}{2}\right)\right),
$$

where $\nu_{r}$ and $\nu_{z}$ count radial and longitudinal excitations, respectively. For given $\nu_{r}$ there are still $g_{\nu_{r}}=\nu_{r}+1$ degenerate states with different numbers of excitations in the two degenerate transversal directions (number of partitions of $\left.\nu_{r}=\nu_{x}+\nu_{y}\right)$. We use the notation $\left(\nu_{r}, \nu_{z}\right)$ for these states.

The $N$-dependence of quantities at zero temperature turns out to have more features than in the isotropic case. For example only in the oblate case $(\lambda>1)$ the notion of shell closures still exists because only then it is energetically favorable to fill up a transversal shell with degeneracy $g_{\nu_{r}}$ before populating a higher longitudinal state. However, these new structures occur on a smaller scale of particle numbers (due to the smaller degeneracy factors) and might be less accessible in experiments with a finite uncertainty of the atom number.

Formulas analogous to Eq. (2.4) can only be given as sums. If $\lambda<1$, one can count all states up to a certain excitation $\left(\alpha_{r}, \alpha_{z}\right)$ by

$$
S_{\alpha_{r}, \alpha_{z}}=\sum_{\nu_{r}=0}^{\alpha_{r}+\left[\alpha_{z} \lambda\right]} g_{\nu_{r}}\left(\left[\frac{\alpha_{r}-\nu_{r}}{\lambda}\right]+\alpha_{z}+1\right)
$$

where $[x]$ denotes the largest integer less than or equal to $x$. Thus, one needs $N=S_{\alpha_{r}, \alpha_{z}}$ particles to populate all states up to $E_{\alpha_{r}, \alpha_{z}}$. For $\lambda>1$, there is an analogous expression. In general, the exact Fermi energy can only be found by searching the lowest state $\left(\nu_{r}, \nu_{z}\right)$ with $N \leq$ $S_{\nu_{r}, \nu_{z}}$. However, the Thomas-Fermi approximation for the Fermi energy is only slightly modified [7]

$$
\tilde{E}_{F}=\hbar \omega(6 N \lambda)^{1 / 3}
$$

The Sommerfeld formula Eq. 2.12) for the chemical potential holds equally in the anisotropic case.

In the following, we give some of the results for anisotropic traps. We concentrate on the case of a heavily deformed cigar-shaped trap, $\lambda \ll 1$ (in fact $\lambda=0.076$ like in [10]). We first consider the dependence of the chemical potential on the temperature in the low-temperature regime. The graph of $\mu(T)$ for 1000 particles displayed in Fig. 7 shows a very intriguing feature: it starts linearly at $T=0$ as predicted by Eq. (2.23) but then goes through a local maximum. The highest occupied state at $T=0$ is $(5,22)$. So $g_{\nu_{r}}=6$ and with Eq. 3.3 $\Delta N=S_{5,22}-N=5$. The next higher state is $(6,9)$. The correction to the linear approximation including this state basically shows that it is responsible for the local maximum. Indeed, the energy difference between the two states corresponds to $k_{B} T=0.157 \hbar \omega \lambda$ which is roughly at the local minimum (cf. arrow in Fig. 7). Thus, at the maximum of $\mu(T)$ the next higher level above the Fermi level becomes thermally accessible and $\mu$ decreases.

As in the isotropic case, the specific heat shows deviations from the Sommerfeld approximation at temperatures where the linear approximation for $\mu(T)$ begins to fail. If $T$ is fixed to a value in that region, the graph of $(6 N \lambda)^{1 / 3} C /(N k)$ as a function of particle number (Fig. 8) exhibits structure on two scales of the particle number, considerably more complex than the isotropic analogue in Fig. 1. The big jumps take place whenever there are enough particles to access a new shell of the 
transversal oscillator. The arrows denote the values $S_{\alpha_{r}, 0}$ for $\alpha_{r}=5,6,7$. Between two such particle numbers (say $\left.S_{\alpha_{r}, 0}, S_{\alpha_{r}+1,0}\right)$ there are 13 major peaks, corresponding to $1 / \lambda \approx 13$ longitudinal states being filled before the next transversal shell can be reached. The finer substructure (see inset of Fig. 8) can also be explained easily: if one starts with the state $\left(\alpha_{r}, 0\right)$ the next state is $\left(0,\left[\alpha_{r} / \lambda\right]+1\right)$ followed by $\left(1,\left[\left(\alpha_{r}-1\right) / \lambda\right]+1\right)$, etc. Consequently, there should be $\alpha_{r}$ maxima before $\left(\alpha_{r}, 1\right)$ is reached. In case $1 / \lambda$ is integer this substructure disappears.

\section{B. Density distributions}

In order to calculate the density distribution we make use of the transverse symmetry and obtain

$\rho(r, z)=2 \sum_{\nu_{r}, \nu_{z}=0}^{\infty} n_{\nu_{r}, \nu_{z}}(T) \sum_{n_{r}=0}^{\left[\nu_{r} / 2\right]}\left|\tilde{u}_{n_{r}, \nu_{r}-2 n_{r}}(r) \cdot \psi_{n_{z}}(z)\right|^{2}$,

where $\tilde{u}_{n_{r}, \nu_{r}-2 n_{r}}(r)$ is the radial wavefunction of a $2 \mathrm{D}$ harmonic oscillator $\left(r^{2}=x^{2}+y^{2}\right)$ with magnetic quantum number $|m|=\nu_{r}-2 n_{r}$ and $\psi_{n_{z}}(z)$ is the wavefunction of the one-dimensional harmonic oscillator. The overall factor 2 allows for the twofold degeneracy of a state with given $\nu_{z}, N_{r},|m| . n_{\nu_{r}, \nu_{z}}(T)$ is the Fermi-Dirac occupation number analogous to Eq. (2.5). The numerical results should again be compared to the ThomasFermi approximation at $T=0$ [7]

$$
\rho(r, z)=\frac{N \lambda}{R_{F}^{3}} \frac{8}{\pi^{2}}\left(1-\frac{r^{2}+\lambda^{2} z^{2}}{R_{F}^{2}}\right)^{3 / 2},
$$

where $R_{F}=(48 N \lambda)^{1 / 6} \sigma$ is the so called "Fermi radius" of the density distribution.

We restrict ourself to a plot of $\rho(r, 0)$ resp. $\rho(0, z)$ (cf. Fig. 9). In transversal direction the density at zero temperature shows only very slight deviations from the Thomas-Fermi result. In contrast, the density in longitudinal direction shown on the right exhibits oscillations around this approximation. These oscillations are mainly due to a step-like behavior of the occupation number $N_{\nu_{z}}$ of the oscillator states in longitudinal direction, which is due to the filling of new transversal states. The steps have a width of $1 / \lambda$. In addition, $N_{\nu_{z}}$ decreases much slower (average derivative $\propto \lambda$ ) than the occupation number $N_{\nu_{x}}$ for the transversal states (average derivative $\propto 1 / \lambda$, here we consider $\rho(x, y=z=0)$ ). As a result, the longitudinal density profile $\rho(0, z)$ receives a bigger contribution from higher states than the transversal one, so one can still see the various maxima of high oscillator states in the first case but not in the latter.

We finally note that for oblate traps the effects are basically the same as in prolate traps. The behavior of the density profiles is interchanged, the abovementioned oscillations occur in the transversal density profile and disappear in the longitudinal direction.

\section{CONCLUSION}

Our calculations have shown that quantum statistical effects on the easily accessible observables of a trapped Fermi gas are restricted to the regime of rather small atom number, e.g. below $N=1000$. For larger atom numbers quantum statistical effects can be more easily understood in terms of a local density approximation [7,11. Our work has been carried out using the grand canonical ensemble. It is known from the ideal Bose gas that the grand canonical and the canonical ensemble give differing predictions [8]. However the deviations are often smaller than the difference between the interacting and the interaction-free case. Problems like artificial fluctuations in a grand canonical ensemble of bosons will not occur for fermions. For that reason we don't dwell on a comparison of the different ensembles here.

The present study of an ideal Fermi gas showed some remarkable effects of the shell structure in the harmonic potential visible e.g. in Fig. 1. For a real Fermi gas the atom-atom interactions will introduce an additional dependence of the chemical potential on the particle number which will smoothen out the steps in Fig. 11. A quantitative prediction of this effect depends on the relative magnitude of atom-atom interactions and the experimentally controllable energy gap $\hbar \omega$. As mentioned in the introduction, an effective suppression of the interactions in the ultracold regime can be attributed to the suppression of s-wave scattering for Fermions in identical substates. Therefore the ideal gas behavior might still be visible provided the harmonic potential is steep enough.

BCS-like behavior as discussed in [4,5] requires two spin states to be trapped. The effects of the harmonic potential in that situation have been allowed for in local density approximation in [5], i.e. for large atom numbers. Because of the sensitivity of the BCS transition to the difference of the atom numbers in both spin states, it however remains a challenge to observe a BCS transition experimentally. By contrast shell effects as discussed in this paper should be visible in a suitable range of small atom numbers and sufficiently large trap frequencies.

\section{ACKNOWLEDGMENTS}

We appreciate stimulating discussions with A. Schenzle and C. Zimmermann. H.W. acknowledges financial support by the DFG under Grant Nr. Wa 727/6. 
[1] M.H. Anderson, J.R. Ensher, M.R. Matthews, C.E. Wieman, and E.A. Cornell, Science 269, 198 (1995).

[2] C.C. Bradley, C.A. Sackett, J.J. Tollet, and R.G. Hulet, Phys. Rev. Lett. 751687 (1995).

[3] K.B. Davis, M.-O. Mewes, M.R. Andrews, N.J. van Druten, D.S. Durfee, D.M. Kurn, and W. Ketterle, Phys. Rev. Lett. 75, 3969 (1995).

[4] H.T.C. Stoof, M. Houbiers, C.A. Sackett, and R.G. Hulet, Phys. Rev. Lett. 7610 (1995).

[5] M. Houbiers, R. Ferwerda, H.T.C. Stoof, W.I. McAlexander, C.A. Sackett, and R.G. Hulet, Report No. condmat/9707006

[6] H.T.C. Stoof, communication at the ESF-Workshop on Bose-Einstein-Condensation, Castelvecchio Pascoli, July 1997.

[7] D.A. Butts and D.S. Rokhsar, Phys. Rev. A 55, 4346 (1997).

[8] The difference between different statistical ensembles for bosons are investigated in H.D. Politzer, Phys. Rev. A 54, 5048 (1996), C. Herzog and M. Olshanii, Phys. Rev. A 55, 3254 (1997) and C. Weiss and M. Wilkens, J. Modern Optics (1997), in press.

[9] J.P. Blaizot and G. Ripka, Quantum Theory of Finite Systems, (MIT Press, Cambridge, 1986), chapt. 6

[10] M.-O. Mewes, M.R. Andrews, N.J. van Druten, D.M. Kurn, D.S. Durfee, C.G. Townsend, and W. Ketterle, Phys. Rev. Lett. 77988 (1996).

[11] J. Oliva, Phys. Rev. B 39, 4204 (1989).

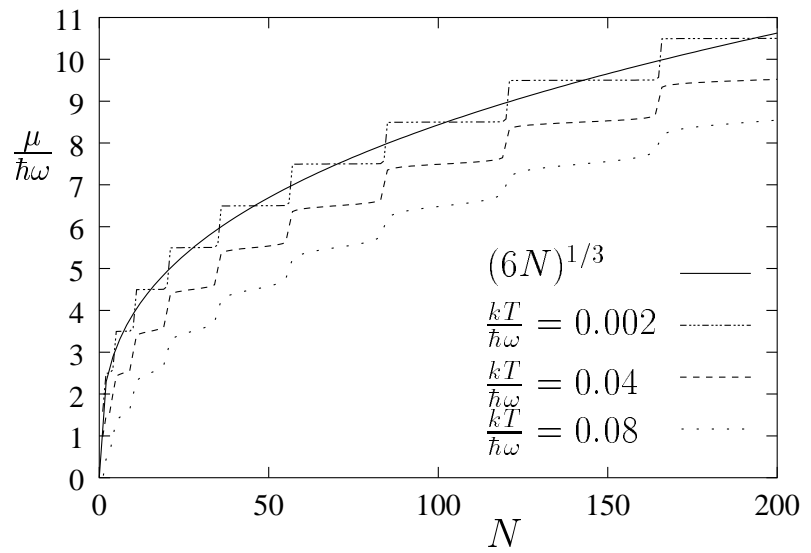

FIG. 1. $\mu(N)$ shows a step-like behavior following the continuous approximation in Eq. (2.11). The dashed and dotted curves are displaced vertically by -1 resp. -2 .

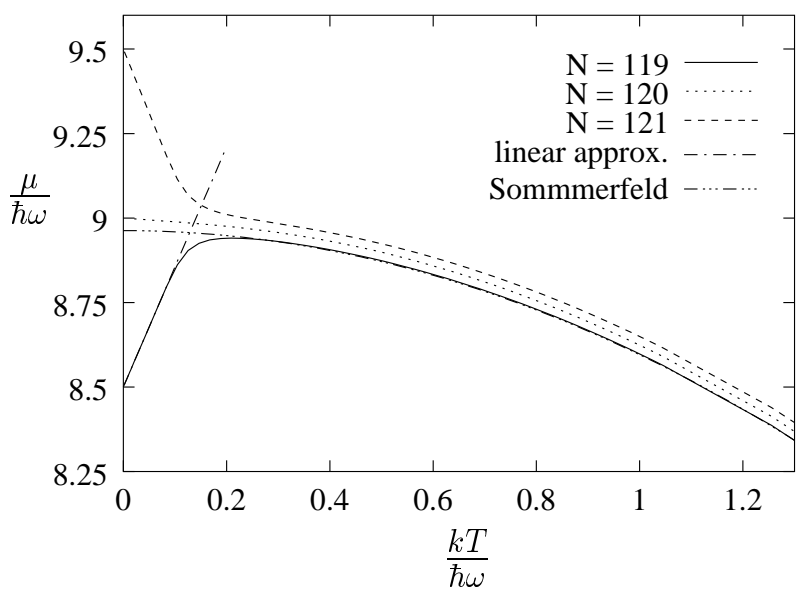

FIG. 2. This figure shows $\mu(T)$ for the isotropic trap. The Sommerfeld approximation is for $N=S_{7}=120$ but agrees very well with the numerical curve for $N=119$. This occurs also for other values of $N$.

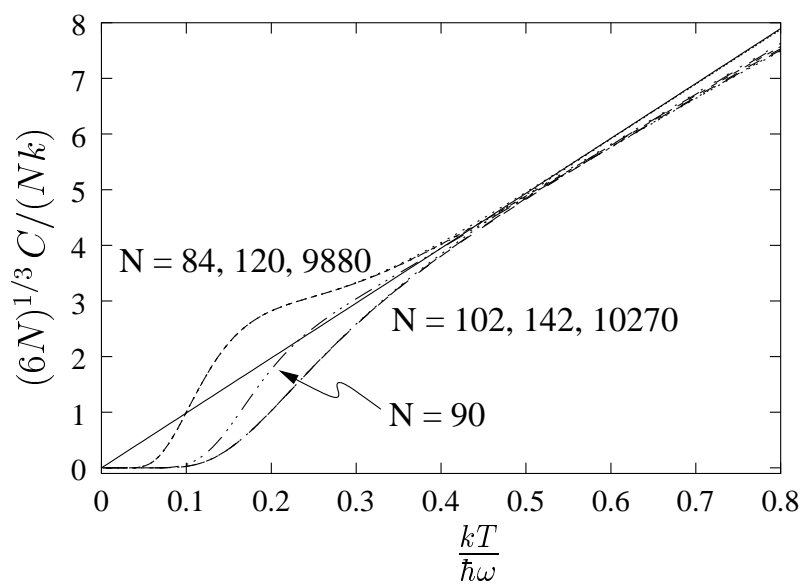

FIG. 3. $C(T)$ appropriately scaled. The linear curve is the Sommerfeld result Eq. (2.27). The deviation at large $k_{B} T / \hbar \omega$ from the linear behavior occurs only for small $N$, because for high temperatures $(6 N)^{1 / 3} C(T) /(N k) \rightarrow 3(6 N)^{1 / 3}$ due to the equipartition theorem.

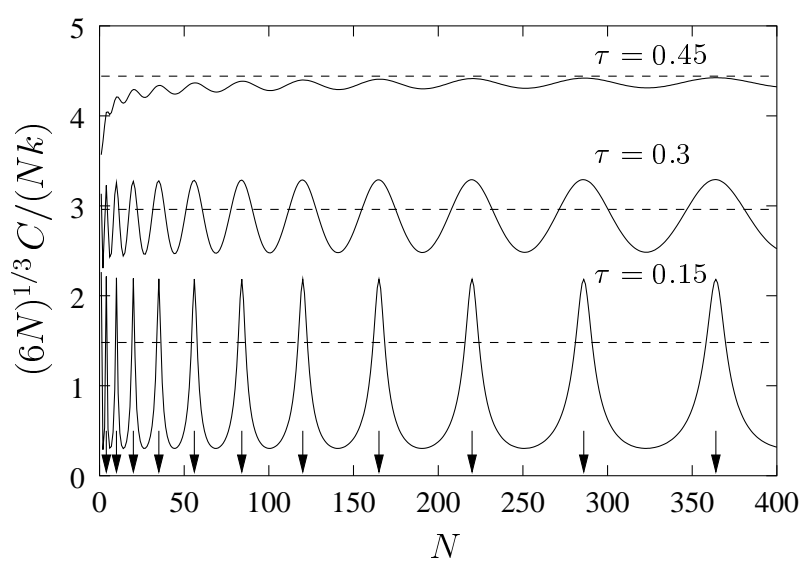


FIG. 4. Specific heat as a function of the particle number at different temperatures and scaled with $(6 N)^{1 / 3}$. $\tau=k_{B} T / \hbar \omega$ denotes the temperature in units of the level spacing. The arrows point to $N=S_{\nu}$ for $\nu=1, \ldots, 11$ $(N=4,10,20,35,56,84,120,165,220,286,364)$.

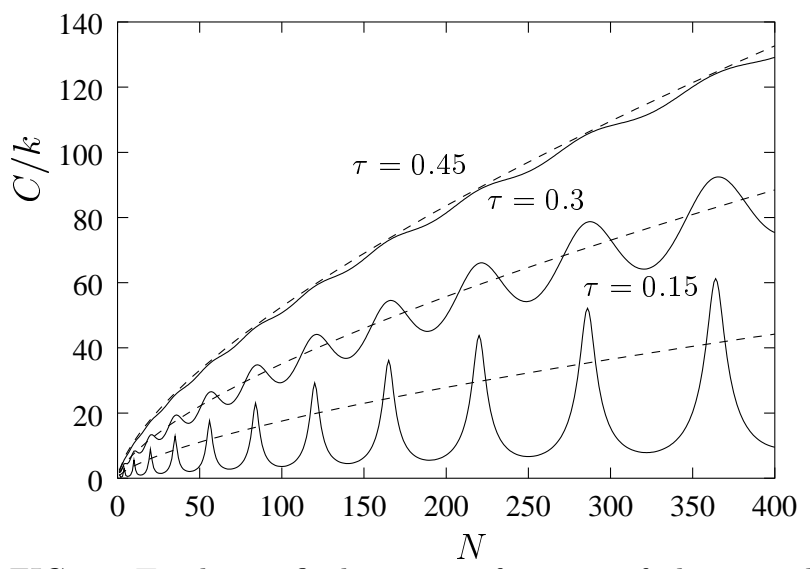

FIG. 5. Total specific heat as a function of the particle number at different temperatures. Again, $\tau=k_{B} T / \hbar \omega$.

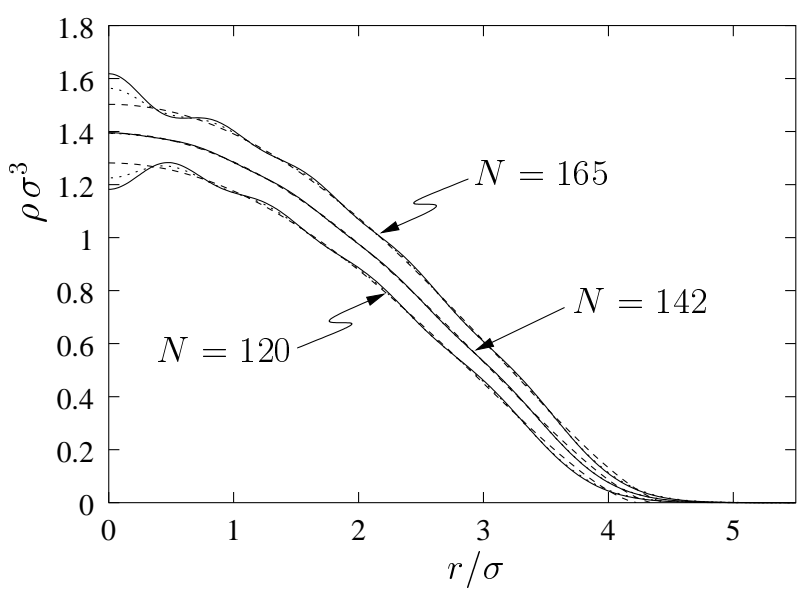

FIG. 6. Spatial density $\rho$ in a isotropic trap as a function of the distance to the trap center for different numbers of fermions. The scaling parameters $\sigma=\sqrt{\hbar /(M \omega)}$ is the width of the ground state. The unbroken lines denote $k_{B} T=0.1 \hbar \omega$, the dotted $k_{B} T=0.25 \hbar \omega$ and the dashed ones are obtained from the Thomas-Fermi approximation at $T=0$.

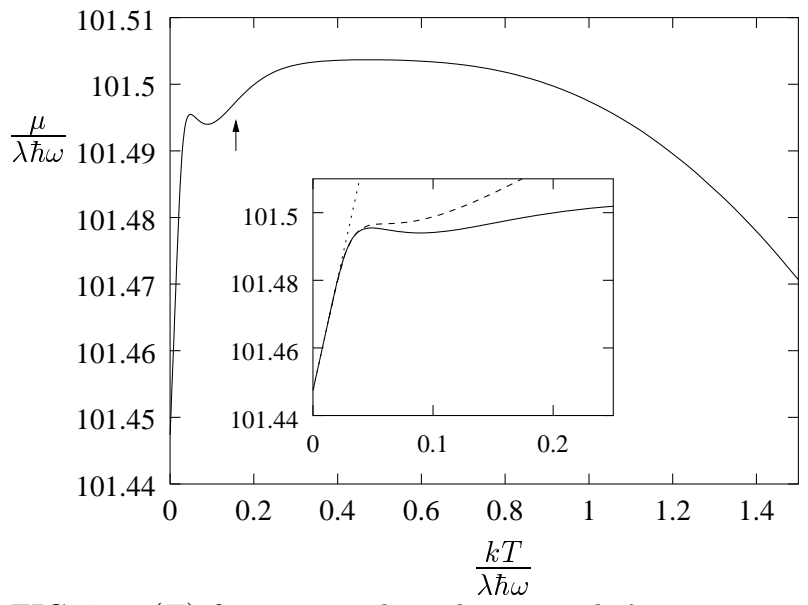

FIG. 7. $\mu(T)$ for a cigar-shaped trap with $\lambda=0.076$ and $N=1000$. The arrow denotes the energy difference between the Fermi level and the next higher state. The inset displays the linear and next higher approximation taking into account the states directly below and above the Fermi level.

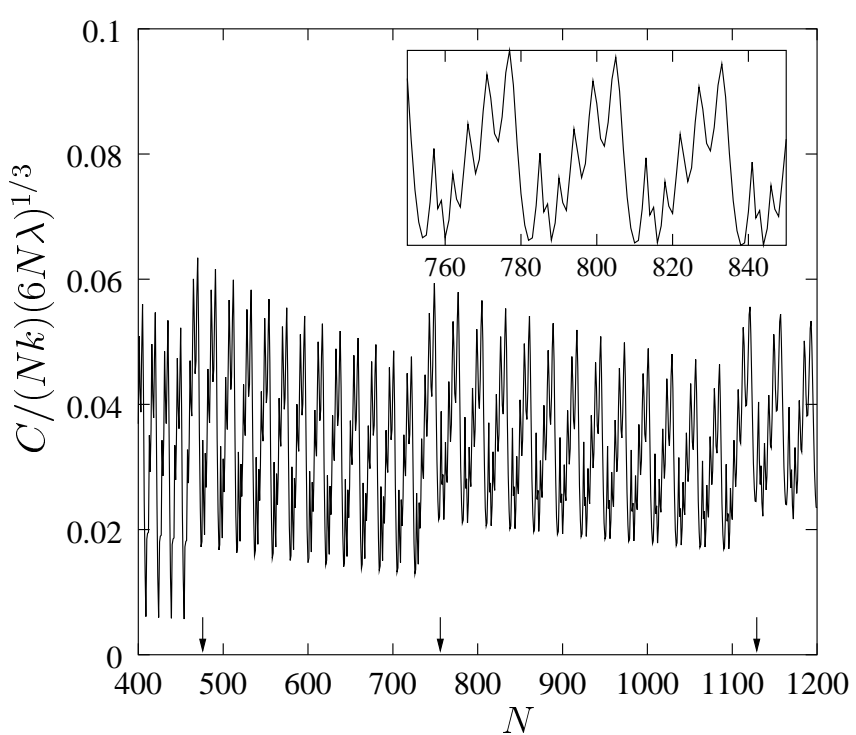

FIG. 8. Specific heat for a cigar-shaped trap as a function of $N$ at $k_{B} T / \hbar \omega=0.044$. The arrows point to $S_{\alpha_{r}, 0}\left(\alpha_{r}=5,6,7\right)$. The inset shows the number range $750<N<850$ in more detail. 


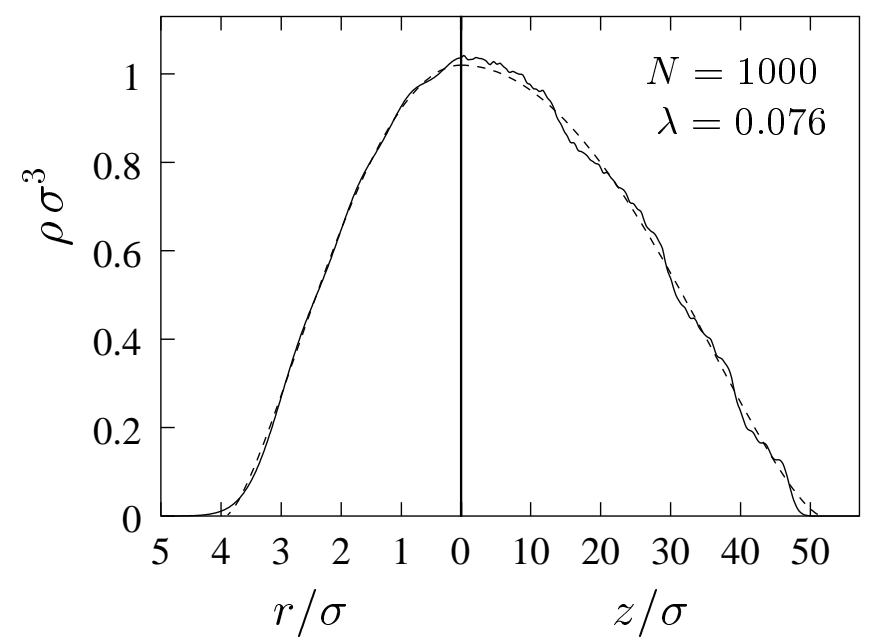

FIG. 9. Density $\rho$ for a cigar-shaped trap. The left part shows the density in transversal direction for $z=0$, the graph on the right is the density in longitudinal direction on the symmetry axis $(x=y=0)$. The unbroken lines are numerical results for $T=0$, the dashed lines come from the Thomas-Fermi approximation. Note that the "Fermi radius" in longitudinal direction is about $1 / \lambda \approx 13$ times larger than the transversal one. 